East African Medical Journal Vol. 86 No. 2 February 2009

DERMATOPHYTE INFECTIONS IN PRIMARY SCHOOL CHILDREN IN KIBERA SLUMS OF NAIROBI

A. Chepchirchir, BSc. (Nursing), MSc. (Med. Microbiology), Tutorial Fellow, School of Nursing Sciences, College of Health Sciences, University of Nairobi, P. O. Box 19676-00202, Nairobi, Kenya, C. Bii, BSc, MSc, PhD, (Medical Mycology), Senior Research Officer, Centre for Microbiology Research, Kenya Medical Research Institute, P. O. Box 47855-00100, Nairobi, Kenya and J. O. Ndinya-Achola, MBChB, MSc, Associate Professor, Department of Medical Microbiology, College of Health Sciences, University of Nairobi, P. O. Box 19676-00202, Nairobi, Kenya

Request for reprints to: A. Chepchirchir, School of Nursing Sciences, College of Health Sciences, University of Nairobi, P. O. Box 19676-00202, Nairobi, Kenya

\title{
DERMATOPHYTE INFECTIONS IN PRIMARY SCHOOL CHILDREN IN KIBERA SLUMS OF NAIROBI
}

\author{
A. CHEPCHIRCHIR, C. BII and J. O. NDINYA-ACHOLA
}

\begin{abstract}
Objective: To determine the prevalence and aetiology of dermatophyte infections in relation to social economic factors in primary school children in Kibera.

Design: A Cross- sectional descriptive study.

Setting: City council sponsored schools namely Olympic, Kibera, Ayany and Mbagathi way all in Kibera, the largest of the informal settlement within Nairobi which is home to between $700,000-1,000,000$ inhabitamts. The study was conducted between September 2006 and February 2007.

Subjects: A total of 422 primary school children from the ages of five years to 15 years were selected for the study.

Results: The prevalence of dermatophytoses was $11.2 \%$ with tinea capitis being the most common type while the grey patch form being the dominant clinical manifestation. There was a significant difference $(p=0.001)$ in dermatophytoses in different schools with Olympic primary school registering the highest prevalence $(22.6 \%)$. The highest infection rate occurred among six to eight years age bracket in both sexes compared to other age brackets $(p=0.002)$. The genera of fungi associated with dermatophytoses were isolated indicating the number in each species as follows; $T$. violecium (35), $T$. mentagrophytes(3), T. terestre(3), T. schoenleinii(2), and T. interdigitale(1), M. canis(2), M. equinum(1) and E. flocossum(1). T. violecium was the predominant species isolated, at 35/48(71\%) followed by T. mentagrophytes and T. terrestre at 3/48 $(6 \%)$ each.

Conclusion: The study indicates high prevalence of $11.2 \%$ dermatophyte infection among the school children in Kibera. Factors contributing to the high frequency and chronic occurrences of ring worm in this area may include poor living environment, children interaction patterns and poor health seeking behaviour. There is need for health education and public awareness campaigns among the communities in urban informal settlements on healthy seeking behaviors and hygiene in order to reduce transmission and severe clinical manifestations.
\end{abstract}

\section{INTRODUCTION}

Dermatophytoses represent a significant public health problem and accounts for almost 19\% of skin diseases affecting school going children in Kenya and especially those from poor socio-economic settings (1).

Dermatological problems manifesting as primary and secondary cutaneous complaints constitute at least $30 \%$ of all outpatient visits to a 
paediatrician (2). In paediatric dermatology, fungal infections of the skin and scalp represent a relatively common problem especially in the tropical and subtropical regions of the world where the warm and humid climates provide a favourable environment for fungi causing superficial mycoses (3).

Tinea capitis is the most common type of dermatophytosis among children aged between six months and pre-pubertal age. Some individuals' manifest with symptoms while others remain asymptomatic. The lack of symptoms in some cases means that the infected individual remain untreated and become reservoirs of infection in close association with the uninfected population $(4,5)$.

Ringworm infection is not a notifiable disease in Kenya but is a cause for concern due to its contagious nature. It can be transmitted through body contacts (person to person) especially in crowded settings or via inanimate objects like cloths, combs or hairdresser equipment and from animals such as household pets (6).

Children before puberty are vulnerably predisposed to dermatophyte infection because of inadequate amounts of inhibitory fatty acids usually produced by an adult's skin (7). Socio-economic predisposing factors include; children's interaction patterns, poor living conditions marked by poor; sanitation, housing (congestion), limited water supply as well as low economic power. Children living in informal settlements are more at risk to such skin diseases spread through contact as a result of living conditions and overcrowding both in school and at home.

Dermatophytosis inflicts a lot of psychosocial trauma due to attached social stigma, ulceration, and sometimes irritation which hampers pupil's concentration in class as well as representing a potential source of secondary bacterial infection. It is not generally appreciated how disabling a skin disease can be since an apparent trivial rash to the observer may be a source of intense discomfort and stigma (8).

Superficial mycoses have neither been the focus of intensive study nor of active control programmes in the sub-Saharan Africa, including Kenya. The neglect is probably due to the fact that fungal diseases of healthy humans tend to be relatively benign (3) Consequently, there is paucity of information on the epidemiology of superficial mycoses in Kenya and this lack of scientific information has negatively affected development of adequate patient management, diagnosis, control programmes and antifungal drug resistance surveillance. Despite the socio-economical improvement in many parts of Kenya, and the efficacy of available antifungal treatment, superficial mycoses remain a common public health problem and a challenge in dermatological practice.
Dermatophytoses is of significantmagnitude and public health problem in informal settlements due to overcrowding and poor environmental sanitation. This report presents the findings of a cross-sectional study of the prevalence of dermatophyte infections among primary school children in Kibera. The public health significance of results and the implications for control of superficial mycoses are discussed and how it affects primary health care delivery in Kenya and other parts of the sub-Saharan Africa with similar setting.

Ethical issues: Informed consent and assent was obtained from the guardians as well as study participants before recruiting them. Approval to carry out the study was obtained from the Kenyatta National Hospital Ethics and Research Committee (ERC) and permission to conduct the study was obtained from City Council's Directorate of Education.

\section{MATERIALS AND METHODS}

Study design:The study was a cross sectionaldescriptive study that focussed on the prevalence, distribution and species differentiation of the causative agents of dermatophytosis in City Council's primary schools in Kibera, Nairobi.

Study area: The study was conducted in Kibera area of Nairobi which has the largest slum settlements in Africa with estimated 700,000 to 1 million residents. It is situated $10 \mathrm{~km}$ south west of Nairobi within Langa'ta division. Informal settlements like Kibera are found in all divisions of Nairobi in varying sizes and densities (9).

Alarger proportion of population in the city livein informal settlements where environmental and health conditions are very poor predisposing the children to communicable diseases like dermatophytoses due to overcrowding both in school and at home (9).

Essential services like health and education are provided by the government through Nairobi City Council, Non-Governmental Organisations, private and faith based organisations.

Most slum houses have small household space with an average room of ten by ten feet being used as a sleeping room, sitting room, bathing room as well as kitchen by household members. Basic amenities like bathrooms and toilets are limited, unsuitable and often communally shared. Water sources are limited with water "kiosks" being the main source of water where a 20 liter jerrican sells for kshs.3.00.(US 0.04) Poor waste disposal creates habitat for rodents and other mammals that could be reservoirs of some fungal elements such as hyphae and spores $(9,10)$.

Kibera has five primary schools within its locality run by the City Council among other privately sponsored schools. City Council sponsored schools 
are affordable for the poor majority compared to the latter. Thus these affordable schools are faced with the problem of congestion and overcrowding due to over enrollment.

Study population: A total of 8176 pupils from the four selected schools constituted the study population. The fifth school, Shadrack Kimalel was used in pilot testing of the study tools. All the schools used for this study therefore were public schools with crowded classrooms and inadequate facilities. Majority of the pupils in these schools are drawn from the expansive Kibera slum and its environments.

Sample size: A total of 422 pupils were selected for the study as estimated using the formula by Andrew et al (10) on sample size calculation. A hypothetical prevalence of $50 \%$ was taken owing to lack of current data in these settings on the above.

Sampling: Purposive sampling was used to select the four city council run primary schools within Kibera. Systematic random sampling method was then used to select the subjects. The desired sample size (n) was 384 while the study population (p) was 8176 The interval of study subjects 'selection was given by $\mathrm{p} / \mathrm{n}(8176 / 385)$ which was 21 . Every $21^{\text {st }}$ pupil was selected. Sampling from each school was done considering the total pupil population: Kibera 116, Ayany 95, Olympic 111 and Mbagathi Way 62.

Inclusion criteria: All pupils who were present in school at the period of study, whose parents consented as well as those who assented to participate in the study.

\section{Study limitations}

(i) Some guardians refused to let their children participate in the study while some pupils had difficulties in expressing information. The researcher and field assistants madeall the efforts to assist the pupils to understand the questions asked and answer them appropriately.

(ii) Braided hair in some pupils concealed the scalp especially with dandruffs increasing suspicion of infection or overlook on existing infection.

(iii) The researcher would have liked to expand the study area to include school children in other informal settlements in Nairobibut due to limited time and resources, this was not possible during the period of study.

Datacollection: A total of 422 pupils wereenrolled from the four schools surveyed and physical assessment was carried out to investigate for presence of dermatophytes infections.

Physical assessment: All the subjects underwent head to toe examination mainly by observation to assess their skin condition and find out if there were any clinical signs of dermatophyte infections. The examination took place at a designated room in each of the selected schools. The socio-demographic data of participants were obtained by interview. To maximise the sense of comfort, female participants were examined by the female member of the research team.

The skin scalp, eyebrows, and eyelashes of each child was carefully examined for characteristic features of tinea capitis according to Orlando and Harmann (12).

Specimen collection: Affected areas were cleansed with $70 \% \mathrm{v} / \mathrm{v}$ ethanol, allowed to dry and light scrapings (skin scales, crusts, hair pieces) were taken from the active edge of lesion using a blunt sterile scalpel blade (10). Pieces of hair stumps were collected in the case of tinea capitis while scrapings from the edges of the lesions were taken in tinea corporis.

Mycological analysis of 160 specimens from suspected infected sites of participants was carried out at the Centre for Microbiology Research, Kenya Medical Research Institute, Nairobi. The specimens were placed in clean white envelopes with each participant's code labeled.

\section{Laboratory procedures}

Direct microscopy: The specimens were examined for presence of characteristics of dermatophyte infection; hyphae or spores, by adding 20\% potassium hydroxide $(\mathrm{KOH})$ and leaving it for 30 minutes for the digestion of keratin to occur. Diagnosis of dermatophytes in the skin scales and crusts was predicted on visualisation through direct microscopy of branching septate hyphae with angular or spherical arthroconidia (arthrospores), usually in chains. Dermatophytes diagnosis in the hair pieces was predicted on visualisation of anthroconidia arranged along the length of the hair in chains or in masses around the hair (ectothrix infection) or in the hair substance endothrix infection (14).

Culture: The specimens were innoculated in Sarborauds Dextrose Agar( SDA) that contains chloramphenical with or without cycloheximide. Both types were used in this study. Chloramphenical inhibits the growth of bacterial contaminants while cycloheximide suppresses saprophytic fungi. Cultures were read weekly to capture pathogens as per their rate of growth. A culture plate was kept for a maximum of eight weeks before being ruled as negative for growth. Identification of dermatophyte species from positive cultures was based on colony characteristics in pure culture and microscopic morphology which includes; the presence of conidia, (macro and micro), and microscopic appearance of the said conidia. 
Physiological tests: Physiological tests were done to aid in species differentiation within a given genus since some share same characteristics. They include urea hydrolysis for T. equinum which doesn't hydrolyse urea, in vitro hair perforation for T. mentagrophytes which gives a positive result and incubation at $36^{\circ} \mathrm{c}$ for T. terestre which grows better at this temperature.

Data management: Data were analysed using SPSS ${ }^{\mathrm{TM}}$. The results were then presented in descriptive statistics using frequency tables, cross tabulation and bar charts. Univariate analysis was used to analyse each variable while bivariate analysis was used to compare dichotomous variables. Frequencies of various parameters were obtained. Chi-square test for significance was used and the level of significance set at 0.05 .

\section{RESULTS}

Atotal of 199 participants were found to havesuspected fungal lesions; $94 \%$ on the scalp (Figure 1), $4 \%$ on the torso(Figure 2) and $2 \%$ on both sites. Tinea capitis was the most common form of dermatophytoses encountered among the pupils investigated, with significant grey patch type of clinicalmanifestation(Figure1) followed by tinea corporis with the circumferential type of lesions (Figure 2). Co-infection of both scalp and torso was least encountered $\mathrm{P}(0.0001)$.

Socio-demographic characteristics of participants: Demographic and socio-economic characteristics are shown in Table 1. All the participants had similar characteristics except for the place of shaving whether at home or in a barber's shop which was statistically significant $\mathrm{P}(0.0001)$.

Table 1

Demographic and socio-economic characteristics of study participants

\begin{tabular}{|c|c|c|c|c|}
\hline & $\begin{array}{c}\text { No. of } \\
\text { participants }\end{array}$ & $\begin{array}{c}\text { Frequency } \\
\text { with } \\
\text { skin infection }\end{array}$ & $\begin{array}{c}\% \text { of total } \\
\text { participants } \\
\text { infected }\end{array}$ & P-value \\
\hline \multicolumn{5}{|l|}{ Age (years) } \\
\hline $6-8$ & 188 & 114 & 60.6 & \\
\hline $9-11$ & 150 & 67 & 44.7 & \\
\hline $12-14$ & 74 & 23 & 31.1 & \\
\hline$<6$ & 8 & 5 & 62.5 & \\
\hline$>14$ & 2 & - & - & 0.039 \\
\hline \multicolumn{5}{|l|}{ Sex } \\
\hline Male & 189 & 81 & 42.9 & \\
\hline Female & 230 & 128 & 55.7 & 0.059 \\
\hline \multicolumn{5}{|l|}{ Next of kin } \\
\hline Parents & 398 & 198 & 49.7 & \\
\hline Relatives & 21 & 11 & 52.4 & 0.181 \\
\hline \multicolumn{5}{|l|}{ Occupation of parent/guardian } \\
\hline Small-scale business & 151 & 65 & 43.0 & \\
\hline Casual jobs & 130 & 69 & 53.1 & \\
\hline Permanent salaried & 75 & 35 & 46.7 & 0.644 \\
\hline \multicolumn{5}{|c|}{ Educational background of guardian/parent } \\
\hline Primary & 78 & 42 & 53.8 & \\
\hline Secondary & 198 & 93 & 47.0 & \\
\hline Tertiary/college & 48 & 23 & 47.9 & 0.944 \\
\hline \multicolumn{5}{|l|}{ Type of living structure } \\
\hline Cemented walls and floors & 213 & 100 & 46.9 & \\
\hline $\begin{array}{l}\text { Cemented floors and } \\
\text { earthen walls }\end{array}$ & 22 & 12 & 54.5 & \\
\hline Mud walled with earthen floors & 162 & 78 & 48.1 & 0.305 \\
\hline
\end{tabular}


Continuation of Table 1

No. of living rooms in house

$1-2$

3-4

$>4$

No. of adults in house

$\begin{array}{lc}1 & 62 \\ 2 & 298 \\ >2 & 63\end{array}$

No. of children in the house

$1-2$

$3-4 \quad 211$

$>5$

101

Sharing

Not sharing

91

No. sharing bed/ beddings

with participant

1

2

$>2$

Source of water for domestic use Piped

Borehole/dam/pond

Stored rain water in tanks

Participants' bathing frequency

At least once daily

Twice daily

Occasionally

Sharing of combs

Family sharing

Not sharing

138

Schoolmates

Sharing

Not sharing

34

390

55

369

$<40$ pupils

$>40$ pupils

Place of shaving hair

Home

49

Barbers' shop

337

132

292

No

Type of pet kept

$\begin{array}{lc}\text { Cat } & 33 \\ \text { Dog } & 95 \\ \text { Both } & 9\end{array}$

$\begin{array}{cc}180 & 50.4 \\ 24 & 44.4 \\ 4 & 40\end{array}$

0.318

34

54.8

47

54

0.753

50

52.6

41.6

0.148

52.3

38.5

0.062

$24-13$

54.2

49.1

$49 \quad 59.0$

0.410

47.8

75

58.3

0.164

47.0

46.7

64.9

0.457

37

52.5

42.8

0.161

35.3

50.5

0.163

12
197

52.7

48.8

0.878

180

34.7

54.9

0.001

185

50.0

$143 \quad 49.0$

0.856

$17 \quad 51.5$

$47 \quad 49.5$

$4 \quad 44.4$

0.959 
Figure 1

Tinea capitis

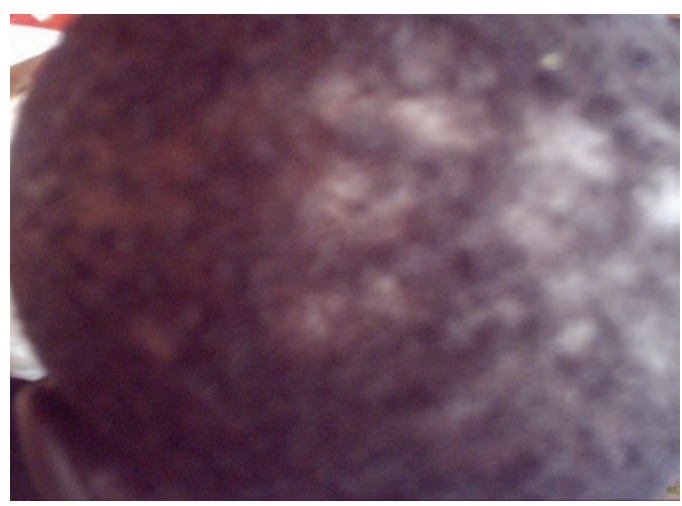

Figure 2

Tinea corporis

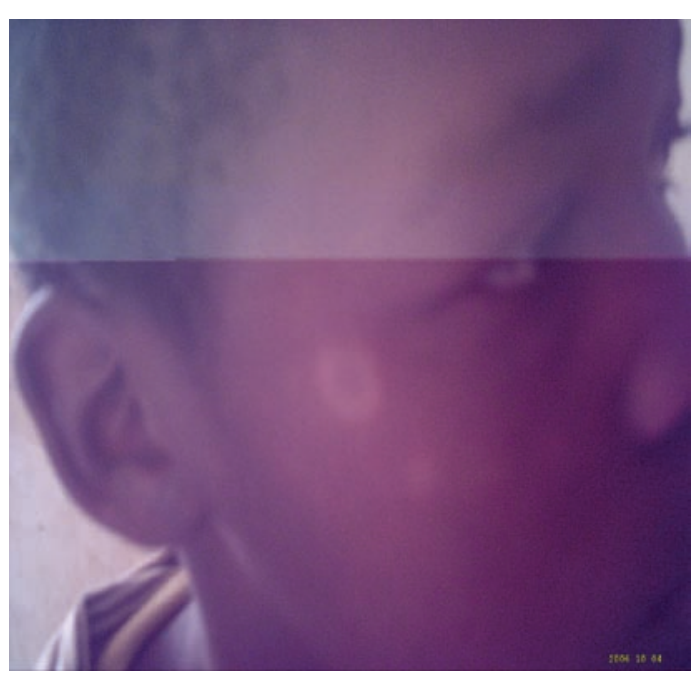

Genus and species of dermatophytes isolated: Based on morphological characteristics and specialised physiologic tests, 48/160 (30\%) specimens were positive for culture. Forty eight dermatophytes comprising nine species belonging to the three geni ; Trichophyton 45/48(93.7\%), Microsporum 2/48(4.2\%) and Epidermophyton $1 / 48(2.1 \%$ ) were isolated (Table 2 ). There was one case of multiple dermatophyte infection by T. schoenleinii and T. violecium. T. violecium species (Figures 3a and b) was the most frequently isolated with 35/48(71\%) followed by T. mentagrophytes and T. shoenleinii (Figures $5 \mathrm{a}$ and $\mathrm{b}$ ) both with 3/48(6.3 $\%$ ). There were two isolates of $M$. canis (Figures $4 \mathrm{a}$ and $b)$.
Appearances of isolated dermatophytes on culture

\section{Figure 3}

T. violecium on SDA+ (Note the violet pigmentation on the media)

3a. Front appearance

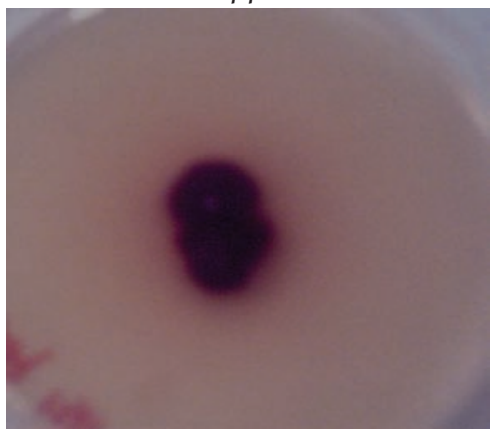

3b. Reverse appearance

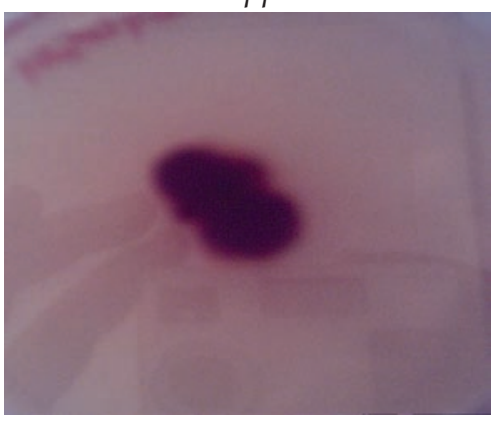

Figure 4

M.canis on mycosel (Note the yellow-brown diffusing pigment)

4a.Front appearance

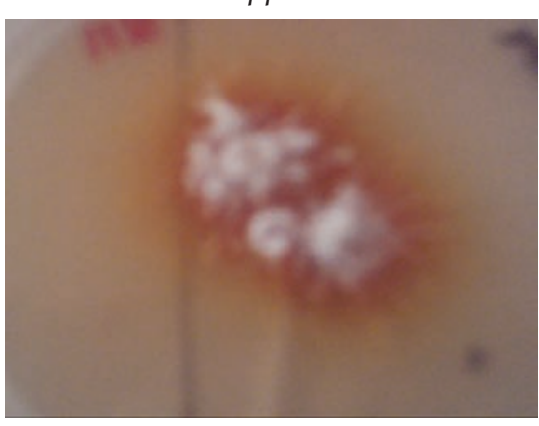

4b. Reverse

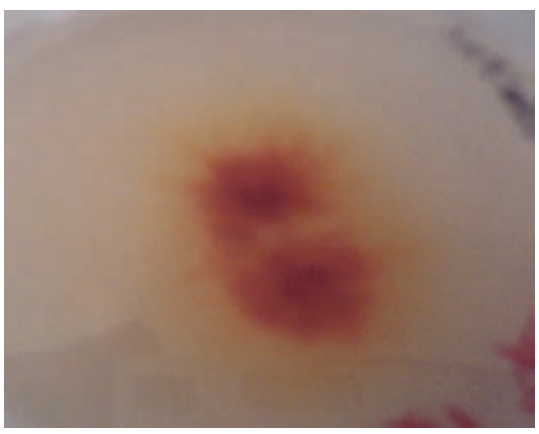


Figure 5

T. shoenleinii on mycosel (Note the white sandy appearance)

5a. Front appearance

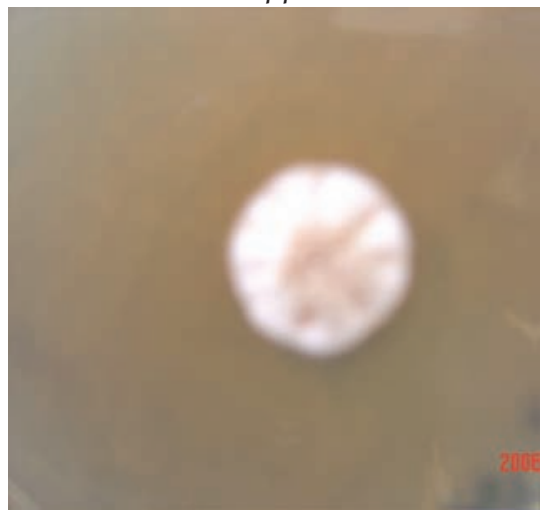

5b.Reverse appearance

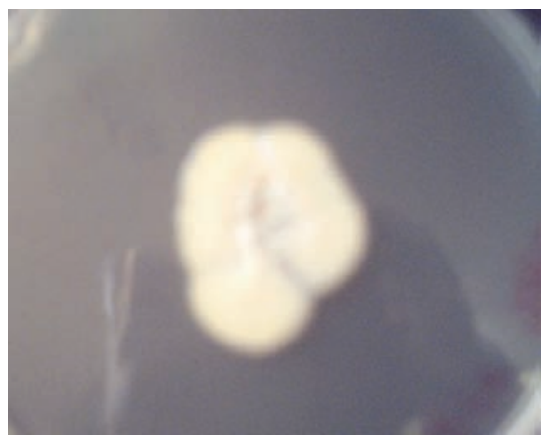

Table 2

Species of dermatophytes isolated

\begin{tabular}{llcc}
\hline Genus & Species & \multicolumn{2}{c}{ Frequency } \\
& & No. & $(\%)$ \\
\hline \multirow{2}{*}{ Trichophyton } & violecium & 35 & 72.9 \\
& shoenleinii & 3 & 6.25 \\
& interdigitale & 1 & 2.1 \\
& mentagrophytes & 3 & 6.25 \\
& concentricum & 1 & 2.1 \\
& terrestre & 2 & 4.2 \\
\cline { 2 - 4 } Microsporum & Sub-total & 45 & 93.8 \\
\cline { 2 - 4 } & canis & 1 & 2.1 \\
& equinum & 1 & 2.1 \\
& Sub-total & 2 & 4.2 \\
Epidermophyton flocossum & 1 & 2.1 \\
& Sub-total & 1 & 2.1 \\
\hline & Total & 48 & 100 \\
\hline
\end{tabular}

\section{DISCUSSION}

The study revealed an overall prevalence of $11.2 \%$ dermatophyte infection among this group of primary school going children. These findings correlate with those of a study done in a rural school in Kisumu whose prevalence was $10.1 \%$ but fell far below the prevalence as per a study done in an urban school in Eldoret with an estimated prevalence of 33.3\% $(15,16)$.

The age range in this study was 6-11 years with the highest number of participants in the 6-8 years group. There was statistically significant difference in the prevalence of infection and the different age categories of the participants $(\mathrm{p}=0.039)$. The infection rates decreased with increase in age with the group of 6-11years being the most susceptible.

Tinea capitis was the most common type of dermatophytoses observed in the participants. This finding concurs with studies done in Kenya, Europe, Asia $(16,17)$. This could be attributed to the exposed nature of the head/scalp compared to other body areas and hence ease of transmission and acquisition of infection. Sharing of hair careequipment, ignorance and poor hygienic practices of hair could be significant factors of contribution. Shaving of hair at a barber's shop was identified as a common phenomenon in urban settings and featured as a significant factor in the study $\mathrm{p}(0.0001)$.

No case of onychomycosis was detected which is in agreement with literature finding that this infection is rare in children (18). The genus trichophyton was the most significant isolate $45 / 48(94 \%)$ while epidermophyton was the least one $(2.1 \%)$ which agrees with other studies done in developing countries in African continent and parts of Asia (19). In the study T. violecium was the most common with $72.9 \%$ dermatophyte species isolated followed by $T$. mentagrophytes $6.3 \%$, M. canis $2.1 \%$, M. equinum $2.1 \%$, T. interdigitale $2.1 \%$, T. terrestre $4.2 \%$, E. flocossum $2.1 \%$, T. schoenleinii $6.3 \%$, and T. concentricum $2.1 \%$. These are anthropophilic species significantly transmitted in overcrowded human settings unlike Europe where most of the dermatophytes are of zoophilic type owing to the high incidence of pet rearing (20).

Studies in Europe have shown that rearing and close proximity to domestic pets is a significant risk factor for the development of dermatophytoses (21). According to this study only a small percentage of households' rear pets as a result of limited space and resources in urban informal settlements. This may be an explanation for the small percentage of zoophilic type of dermatophytes isolated in this study especially those associated with domestic pets like M. catis and M. canis. 
Poor garbage disposal has been associated with the presence of zoophilic type of dermatophytes owing to the presence of rodents that is, rats (22). This is a common phenomenon in slum characterised by heaps of garbage around living houses. Rodents scavenging into living houses in search of food may be reservoirs for fungal pathogens. Such mammals harbor dermatophytes like T. mentagrophytes which was a significant isolate in thisstudy. T. mentagrophytes was the second most prevalent species isolated. No geophilic type of dermatophyte was isolated in the study. Although this could not be clearly explained, this could be attributed to hygiene standards and universal dressing which limits the body's contact with the contaminated soil.

Most of the infected children were aged below ten years affirming the observation that a ringworm infection is predominantly a pre-pubertal disease. This is confounded by poor hygiene at this age as well as the absence of saturated fatty acids that provide a natural protective mechanism against dermatophytoses. Besides this natural predisposition, the degree of exposure to aetiological agents plays a major role. Childrenbroughtup in clean environments with less crowding and reliable water supply tend to suffer less from dermatophytes (23).

A similar prevalence rate of infection among girls and boys was noted in the study contradictory to other reports elsewhere indicating that boys were more vulnerable. This may be an isolated finding but could be an indication of possible change in the social behaviours of the participants $(15,16)$.

No major complication of dermatophytosis was detected in the infected population except for two cases who had secondary bacterial infections marked by suppurative lesions on the scalp. The bacterial agent in the infection was identified as Staphylococcus aureus and was commonly seen with zoophilic type of dermatophytes which were significantly few in the study.

Most children put up with their biological parents which contradicts the report on population and health dynamics in Nairobi's informal settlements which indicates that a higher proportion of resident children under the age of 15 years are not the biological offspring of the head of household implying a higher fostering rate (9).

The level of education of parents and guardian's had no significance with the presence of dermatophyte infection in children in the study. Most parents had secondary level of education which probably enables them have a good understanding of good public health practices. The carriage and spread of ringworm infection is dependent on the interaction patterns and living conditions. Most parents in slum settings cannot afford less crowded schools for their children nor afford decent living conditions due to underlying economic constraints which could significantly influence transmission of dermatological conditions. The form of employment had no significance in risk of dermatophytoses in the study. Most parents had low paying jobs regardless of whether they were on permanent or casual basis. In the urban areas, the economic power determines where one lives which in turn dictates the potential exposure to fungal agents.

In the study, most of the participants' accessed piped water through water "kiosks" or sometimes from water vendors whenever there is some shortage. This is characteristic of most slums with distinct lack of basic amenities like electricity, proper sanitation and access to piped water (9).

Most of the participants' living structures were semi- permanent with cemented floors and mud walls. However the type of living structure was not a significant factor for dermatophyte infections among children in these settings. This is probably attributed to fairly good hygiene standards unlike that of rural settings.

Hygiene status of an individual's skin has been cited as one of the risk factors associated with the acquisition of dermatophyteinfections. The poorer the hygiene, the higher the chances of acquiring infection. Over $75 \%$ of the participants reported bathing at least once daily. High frequency of bathing reduces rate of colonisation of the skin by the fungal agents following contact with infective agents.

In the study, it was noted that children in the same class had similar type of infection which could be attributed to contagion associated with overcrowding and proximity among the pupils. It has statistical significance at $\mathrm{P}=0.001$. Sharing of beddings and toiletries was noted as a common practice among the participants at home. On average two people in the participants homes shared beds. Over $66 \%$ of participants shared bath towels and combs with family members while $78.5 \%$ participants shared beds and beddings. Over $68 \%$ of the participants shared beds with two bedmates while $8 \%$ shared with three bedmates. However there was no significant difference between the number of family members sharing and the occurrence of skin infections $(\mathrm{p}=0.161)$.

Skin infections can affect ones' self esteem especially children and may interfere withindividuals' performance despite adequate potential to excel; although the presence of dermatological condition had no significance on the participant's performance in examinations.

General discrimination against those with dermatophyte infections affected was noted in 54\% of the respondents. However discrimination had no 
significant influence on academic performance of the infected. This may be due to more efforts directed on academics with limited time on socialisation as well as children's ability to forgive and forget. Although it was not significant academically, it is significant from a social perspective in terms of the individuals' self regard and social development which might be affected irreversibly.

In the study, 96/186(51.5\%) of infected pupils had had the infection for six months and those who sought treatment, 63/186(34\%), used it for less than two weeks increasing the likelihood of resistance and transmission. Most of the participants reported having used topical antifungal agents. These may not be effective in the course of widespread infection and especially in treatment of endothrix type of infection. Oral systemic antifungal agents like Griseofulvin are recommended for widespread and chronic dermatophytosis for effective management. Seeking for treatment alone is not adequate but rather adherence to the mode and frequency of treatment. Shorter treatment period not only contributes to re-emergence of similar infections but promotes development of antifungal drug resistance.

Prompt and lengthy period of treatment of fungal infections of the skin is recommended. Only $54 \%$ sought for treatment with only $22 \%$ completing the treatment for the recommended period of three to four weeks.

One of the greatest challenges of prevention and eradication of dermatophytoses is the presence of healthy asymptomatic carriers. This is confounded by inadequate treatment marked by shorter periods of topical antifungal use as well negligible use of oral treatment. This approach results in disappearance of symptoms but persistence of infection resulting in high recurrence rate and prolonged carriage. Parent's/ guardian's ignorance, on the importance of completing prescribed doses of drugs and health seeking behaviour is an important factor in management of dermatophytoses.

The presence of spores on $\mathrm{KOH}$ preparation was significant indication of the presence of dermatophyte infection and outcome on culture $(\mathrm{P}=$ 0.0001 ). Although $\mathrm{KOH}^{\prime}$ s reliability as a preliminary diagnostic technique for dermatophytes is low, it is a cost effective procedure in resource constrained set-ups but acts as a supportive preliminary investigation.

In conclusion the prevalence of dermatophyte infections among children was 1.2\% higher than the supposed rate of $10 \%$ with tinea capitis being the most common clinical type. Communal shaving was noted as a significant risk factor. No difference in infection burden was observed between boys and girls. This reflects a change in the pattern of these infections usually taught to affect boys more than girls. The younger age groups were more susceptible than the older age groups thus need for health education targeting them. Upgrading of theinformal settlements will go along way in improving the living conditions of the affected.

\section{ACKNOWLEDGEMENTS}

To Kenya Medical Research Institute for allowing use of their laboratory facilities in processing of mycology specimens; M. Olga, technical bench assistant, Mycology laboratory at Kenya Medical Research institute; Mr. F. L. Song'ole of City Council of Nairobi's Directorate of Education; Research assistants for their time and dedication; Captain M. Ngui for entering data, D. Kimeli for analysing data and professional advisers, Merrs P.M. Waithaka, A.A. Ayieko, J. Mwaura and L. Nyabola, all lecturers at the University of Nairobi.

\section{REFERENCES}

1. F. Edhonu-Elyetu. Communicable ectoparasitic and fungal skin diseases among five to fifteen year old children in Kaloleni Division, Kilifi District, Coast Province, Kenya. MPH thesis, University of Nairobi. 1991.

2. Thappa, D.M. Common skin problems. Indian J. Pediatr. 2002; 69:701-706.

3. Shrum, J.P., Millikan, L.E. and Bataineh, O. Superficial fungal infections in the tropics. Dermatol. Clinic. 1994; 12: 687-693.

4. Ive, F.A. The carrier stage of tinea capitis in Nigeria. Brit. J. Dermatol. 1966,78: 219-221.

5. Yehia, M.M. Studies on dermatophytes in Mosul and vicinity [Thesis]. Mosul, University of Mosul, College of Medicine, 1980; 48-106.

6. Figueroa, J. I., Hawraneck, T., Abraha, A. and Hay, R. J. Tinea capitis in south western Ethiopia: a study of risk factors for infection and carriage. Int. J. Dermatol. 1997; 36:661-666.

7. David, G., Richard, C. B., et al. Medical Microbiology: a guide to microbial infections, pathogenesis, immunity, laboratory diagnosis and control. Churchill Livingstone. 16th edition: 2003. pg 568-579.

8. Bibeka, N.J., Vijay, K. G., Agrawal, S., et al. Tinea capitis in Eastern Nepal. Int. J. Dermatol. 2006; 45: 100-103.

9. Matrix development consultants 1993, World Bank 1999.

10. Andrew, A., Fisher, J., E., Laing, J. E., et al. Handbook for Family Planning Operations Research Design. Second Edition. 1998. The population council. Pg. 43.

11. Elewski, B.E. Tinea capitis: a current perspective. J. Amer. Academy of Dermatol. 2000; 42:1-20. 
12. Orlando C. and Harmann M. Clinical Tropical Dermatology, WB Saunders. 1996. 1st Edition. page 12-13.

13. Ogunbiyi, A.O., Owoaje, E. and Ndahi, A. Prevalence of skin disorders in school children in Ibadan, Nigeria. Pediatric Dermat. 2005; 22: 6-10.

14. Ayaya, S. O., Kamar, K.K. and Rakai, R. Etiology of Tinea capitis in school children. East Afr. Med. J. 2001; 78: 531-535.

15. Schmeller, W., Baumgartner, S. and Dzikus, A. Dermatophytomycoses in children in rural Kenya: Impact of Primary Health Care. Mycoses. 1997; 40: 55-63.

16. Cuetarama, M. S., Del Palacio, A., Pereiro, M., et al. Prevalence of undetected Tinea capitis in a school survey in Spain. Mycoses. 1997; 40:131-134.

17. Jahangir, M.,Dunwell ,P., Barret R., D. and Rainford, L. Clinical-etiologic correlation in Tinea capitis. Int. J. Dermatol. 1999; 38:275-278.
18. Gawk, R.D. Illustrated Color Text of Dermatology. Longman Group of Publishers: 1992; pg.574-63I.

19. AI Mutahiri, N., Arun, J., Amr, Z., et al. Tinea capitis among children and adolescents in the Farwaniya region of Kuwait. J. Dermatol. 2003; 30: 904-906.

20. Trivino-Duran, L., Torres-Rodriquez, J.M., MartinezR. Prevalence of Tinea capitis and Tinea pedis in Barcelona school children. Pediatric Infect. Dis. J. 2005; 24:137141.

21. Collee, J.G., Fraser, A.G., Dugid, J. G., et al. Practical Medical Microbiology. 1996. Churchill Livingstone Publishers. 14th edition: 703-717.

22 Suzanne, C.O.S., Brenda, G. B., Kerry, H. C., et al. Textbook of Medical Surgical Nursing, J.B Lippincott Company. 5th Edition: 1984. pg. 955-956.

23. Mark, H. B., Andrew, J. F., Thomas, V. J., et al. The Merck Manual of Medical information. Merck \& Co. 4th Edition: 1999; pg 979-980. 